Bull. Mater. Sci., Vol. 6, No. 6, December 1984, pp. 1075-1086. (C) Printed in India.

\title{
Electrical properties of films at microwave frequencies
}

\author{
D C DUBE \\ Physics Department, Indian Institute of Technology, New Delhi 110016, India \\ MS received 1 June 1983
}

\begin{abstract}
A technique suitable for the measurement of electrical properties of films/sheets at microwave frequencies has been described. Groups of materials are identified for which the absence of any kind of electrodes in these microwave measurements can be utilised with advantage. Results are presented for a variety of materials such as metal films, superionic conductors, semiconductors and insulating polymers.
\end{abstract}

Keywords. Microwaves; waveguide; dielectric constant; a.c. conductivity.

\section{Introduction}

Knowledge of the electrical properties of films at microwave frequencies is helpful in material study and characterization for device fabrication. Thin metal and insulating films, for example, are extensively used in microwave integrated circuits. There, it is of interest to know the attenuation and phase produced by a particular film and variation of these quantities with parameters such as film thickness, temperature etc. Thin semimetal and metal films were studied by several workers (Gunn 1967; D'Aiello and Freedman 1969; Duggal and Rup 1969; Ramey et al 1970). The microwave applications of thin metallic films have also gained importance as exemplified by the use of films as microwave mirrors and as large size film reflectors (Korolev and Gridnev 1963). Microwave measurements are essential in the above cases. But in many situations advantage can be taken of the fact that at microwave frequencies the measurements basically are electrodeless and therefore these are recommended to study materials which require special type of electrodes. Groups of materials may be identified where contacts do pose problems, such as e.g. (i) semi-conductors (ii) fast ion conducting solids (iii) membranes and other biological systems. Such materials can be conveniently studied at microwave frequencies.

Section 2 describes few techniques including the one developed earlier (Dube and Natarajan 1973), for the measurement of dielectric constant, loss and conductivity of films and toils. Finally the results are presented for a variety of films.

\section{Measurement techniques}

The most widely used method for microwave measurements of dielectric parameters of solids is the short-circuited line technique of Von Hippel (Von Hippel 1961). The technique involves the measurement of vswR (voltage standing wave ratio $=E_{\max } / E_{\min }$ ) on the standing waves set-up when the microwaves are totally reflected from a short circuit. A dielectric specimen in the form of a small block which completely 

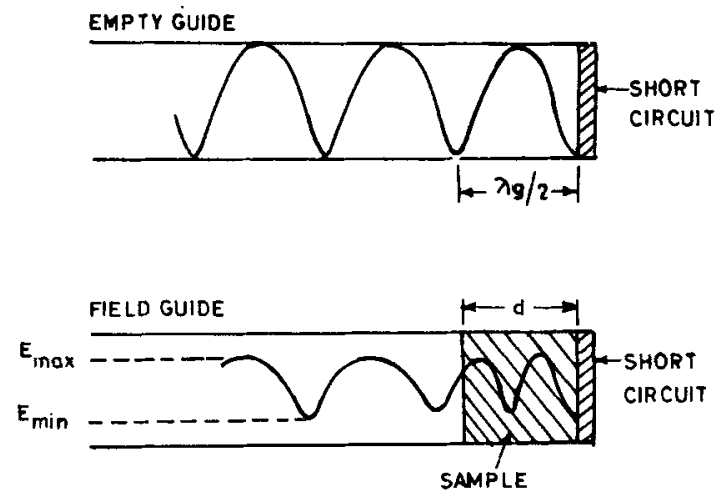

Figure 1. Standing wave pattern in empty and field guide.

fills the section of waveguide is placed next to the short circuit. This alters both the position of standing wave pattern and the size of the voltage ratio by amounts which depend on the dielectric constant and loss of the material and on the length of the 'filled guide' (figure 1).

Von Hippel's method when applied to a very thin specimen does not give accurate results, the reason being the lack of strong interaction between the e.m. wave and the thin specimen. Therefore the presence of the specimen shifts the voltage minima only slightly (often insignificantly) and the change in standing wave ratio is also small (often not measurable accurately).

Recently, Von Hippel's technique has been modified to suit thin samples by measuring impedance not only with short circuit (figure 1) but also using an open circuit and matched terminations (Das Gupta and Pramanic 1982). The method is effective only for high dielectric constant films.

Cavity methods which measure the change in resonance frequency and broadening of resonance response on introduction of the specimen are also not very successful for the same reasons of lack of strong interaction when the specimen is very thin. However, attempts have been made to study thin samples using specific forms of cavities (Conklin 1965; Sobol and Hughes 1967) and other forms of sample holders (Pilipenko et al 1970). Cavity perturbation technique has also been used (Rzepecka and Hamid 1972).

\section{An accurate and convenient technique}

In the above techniques such as that of Von Hippel, the accuracy of measurement is poor due to weak interaction of e.m. wave with the specimen. And the reason for the poor interaction is the fact that in normally used $\mathrm{TE}_{10}$ mode of excitation the electric field is maximum at the centre of the waveguide and diminishes to zero at the side walls (figure 2); most of the portion of the specimen lies in the weak field regions. Keeping this in view, a suitable technique was developed (Dube and Natarajan 1973) to study thin film specimens. In this technique the thin specimen is placed longitudinally at the centre of the broad side of a hollow rectangular waveguide excited in the $T E_{10}$ mode so that the whole specimen remains in maximum electric field as shown in figure 3 . In this geometry Maxwell's equations can be solved for the $\mathrm{TE}_{10}$ mode by taking $Z$ variations of the form $\exp (-i \beta Z)$ (axis of waveguide is along the $z$-direction). Then by usual 

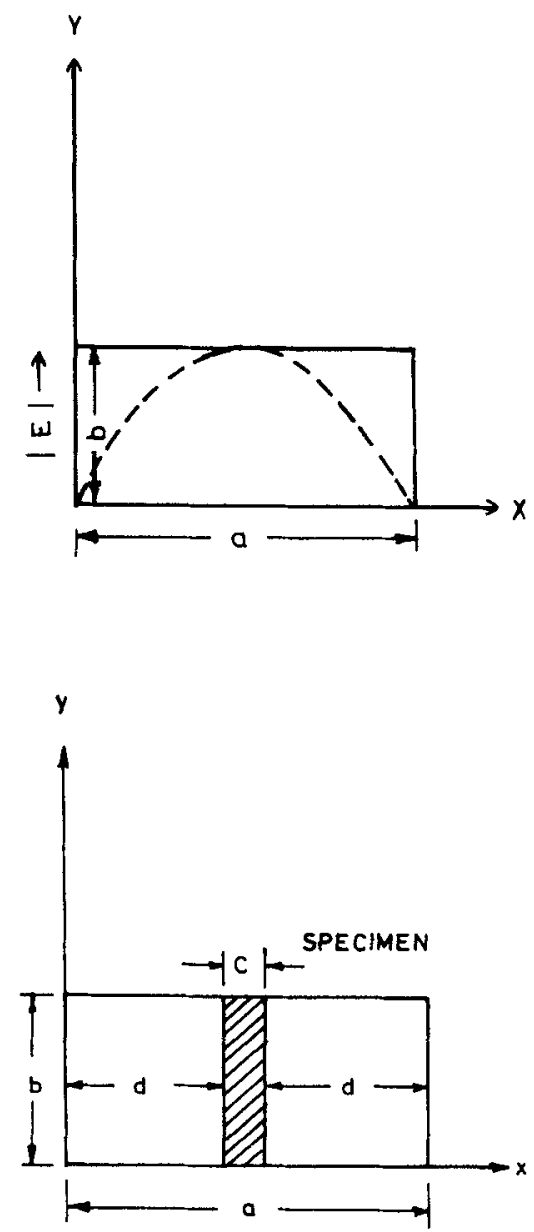

Figure 2. Electric field distribution in a rectangular waveguide in the $T E_{10}$ mode.

Figure 3. Cross-section of a rectangular waveguide with specimen.

methods (Collin 1960), the following dispersion relation is obtained

$$
\tan \left[d\left(k^{2}-\beta^{2}\right)^{1 / 2}\right] \tan \left[\frac{1}{2} c\left(\varepsilon k^{2}-\beta^{2}\right)^{1 / 2}\right]=\frac{\left(k^{2}-\beta^{2}\right)^{1 / 2}}{\left(\varepsilon k^{2}-\beta^{2}\right)^{1 / 2}}
$$

where $k=\omega / C_{0}$ is the free space propagation vector and $\omega$ is the angular frequency. Equation 1 is a transcendental equation, and its solution can be obtained only by graphical methods. For complex dielectric constants, the solution of (1) is very difficult even graphically. Therefore, we simplify (1) under the approximation $\frac{1}{2} c\left(\varepsilon k^{2}-\beta^{2}\right)^{1 / 2}<1$, which holds very well in the case of thin films; hence using $\varepsilon=\varepsilon_{r}-i \varepsilon_{i}$ and $\beta=\beta_{r}-i \beta_{i}$, we get the following expressions for the real and imaginary parts of the complex dielectric constant:

$$
\begin{aligned}
& \varepsilon_{r}=\frac{1}{k^{2}}\left(\left(\beta_{r}^{2}-\beta_{i}^{2}\right)+\frac{a_{1} a_{3}+a_{2} a_{4}}{a_{3}^{2}+a_{4}^{2}} \cdot \frac{1}{\frac{1}{2} c d}\right), \\
& \varepsilon_{i}=\frac{1}{k^{2}}\left(2 \beta_{r} \beta_{i}-\frac{a_{2} a_{3}-a_{1} a_{4}}{a_{3}^{2}+a_{4}^{2}} \cdot \frac{1}{\frac{1}{2} c d}\right),
\end{aligned}
$$


where

and

$$
\begin{aligned}
& a_{1}=d \operatorname{Re}\left(k^{2}-\beta^{2}\right)^{1 / 2} \\
& a_{2}=d \operatorname{Im}\left(k^{2}-\beta^{2}\right)^{1 / 2} \\
& a_{3}=\frac{2 \sin 2 a_{1}}{\exp \left(2 a_{2}\right)+\exp \left(-2 a_{2}\right)+2 \cos 2 a_{1}}
\end{aligned}
$$

and

$$
a_{4}=\frac{\exp \left(2 a_{2}\right)-\exp \left(-2 a_{2}\right)}{\exp \left(2 a_{2}\right)+\exp \left(-2 a_{2}\right)+2 \cos 2 a_{1}}
$$

$\beta_{i}=$ attenuation introduced by unit length of the specimen (measured in nepers $\mathrm{m}^{-1}$ )

$\beta_{r}=\beta_{p}+\beta_{0}$ (measured in radians $\mathrm{m}^{-1}$ )

where

$\beta_{p}=$ phase shift produced by the unit length of the specimen

and

$\beta_{0}=2 \pi / \lambda g$, where $\lambda g$ is the guide wavelength in the empty guide,

$c=$ thickness of specimen, and

$d=\frac{1}{2}(a-c), a$ being the width of the waveguide (figure 3 ).

Thus, in complex dielectrics, the value of $\varepsilon_{r}$ and $\varepsilon_{i}$ can be obtained from (2) and (3). For loss-free dielectrics the solution of (1) can be obtained without any approximations. The two main quantities required to be measured experimentally are the phase and attenuation introduced by the specimen. Two experimental arrangements have been proposed (Dube and Natarajan 1973, 1974). Either may be used for these measurements. Both make use of rectangular waveguide system excited in the $\mathrm{TE}_{10}$ mode. One arrangement has been shown in figure 4.

Microwave power is obtained from a Klystron source. The power is divided into two arms at the 10-dB directional coupler. The lower arm contains the standard impedance, i.e., a standard variable attenuator and a standard variable phase shifter $\left(0^{\circ} \rightarrow 360^{\circ}\right)$. The upper arm contains the sample holder which is an approximately $10 \mathrm{~cm}$ long waveguide having a sharply cut longitudinal slot at the middle. The sample is introduced through this slot. The powers from the upper and the lower arms are again combined at a 3-dB directional coupler. The power output from the directional coupler is fed to the detecting system.

To start with, a null point is obtained in the detector by properly adjusting the variable attenuator and the phase shifter. The introduction of the specimen disturbs the balance. The null conditions are restored by varying the attenuator and phase shifter. Thus the phase shift and attenuation introduced by the sample can be accurately measured. Large sample lengths should be used for producing large phase shifts and attenuations.

Reflections from the edge of the sample are normally extremely small. But these can be detected by a separate experiment and if required, tuner no. 8 in figure 4 may be used for matching purposes.

The minimum detectable phase shift and attenuation in the measuring system were $0.25^{\circ}$ and $0.02 \mathrm{~dB}$, respectively. 


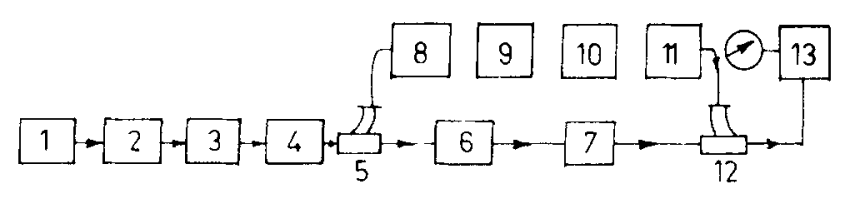

Figure 4. Schematic diagram of the experimental set up: 1. Klystron power source; 2. Frequency meter; 3. Padder attenuator: 4. Ferrite isolator; 5. and 12, Directional couplers; 6. Calibrated variable phase shifter; 8 and 11 tuners; 9. Variable attenuator; 10 . sample holder waveguide; 13. Amplifier and meter.

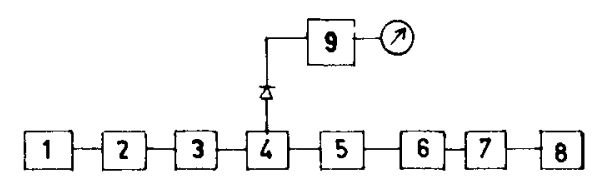

Figure 5. Schematic diagram of the experimental set-up; 1. Klystron power source; 2. Frequency meter; 3. Ferrite isolator; 4. Waveguide travelling detector; 5 . variable attenuator; 6. E-H tuner; 7. sample holder waveguide; 8. movable short; 9. Amplifier and meter.

To study dielectric parameters as a function of temperature, the guide section containing the specimen may be cooled by a low temperature bath around the section or may be heated by a cylindrical furnace.

The other experimental set-up (Dube and Natarajan 1974) is shown in figure 5. This is a simpler arrangement and does not require calibrated variable phase shifter and attenuator and is as accurate as the first set-up.

Here the travelling wave detector is used to measure the shift of the voltage minimum when the specimen is introduced. Let the specimen length be $L \mathrm{~cm}$ and the phase shift produced be $X \mathrm{~cm}$, then phase introduced by the specimen per unit length is

$$
\beta_{s}=\beta_{0} \frac{X}{L}
$$

Thus the total phase constant $\beta_{r}$ is given by

$$
\begin{aligned}
& \beta_{r}=\beta_{0}+\beta_{s} \\
\text { or } \quad & \beta_{r}=\beta_{0}\left(1+\frac{X}{L}\right)
\end{aligned}
$$

For measuring the dielectric loss, the vswr is taken with and without the specimen. Let these be $\rho_{1}$ and $\rho_{2}$; then

$$
r=\frac{\rho_{1}-1}{\rho_{1}+1}=\exp (-\alpha)
$$

and

$$
r^{\prime}=\frac{\rho_{2}-1}{\rho_{2}+1}=\exp -\left(\alpha+2 \beta_{i} L\right)
$$

where $r$ and $r^{\prime}$ are the reflection coefficients of the system with and without the sample and $\alpha$ is the attenuation constant. Hence $\beta_{i}$ can be calculated from (4) and (5). 


\section{Results and discussion}

The accuracy of the proposed technique for measuring the dielectric parameters of films was checked by studying two materials namely, myler and teflon whose dielectric properties are well known over a wide frequency range including microwaves. Results are summarised in table 1.

In the recent past the technique has been used to study films/sheets/wafers of varying nature and differing widely in electrical properties. Some of the results are given below.

Complex permittivity and conductivity have been studied for silicon semiconductor (Natarajan et al 1978). $\varepsilon_{r}$ and $\varepsilon_{i}$ values for one sample of $p$-silicon for various thicknesses are given in table 2 . The thickness of the starting sample was reduced by etching and lapping.

The d.c. conductivities measured by the four-probe technique and the microwave conductivities obtained from the relation $\sigma_{\text {a.c. }}=\omega \varepsilon_{0} \varepsilon_{i}$ are compared in table 3 for three samples of p-silicon.

The microwave conductivity is slightly less than the d.c. values. This is somewhat

Table 1. Dielectric parameters of teflon and mylar films at $9.375 \mathrm{GHz}$ at room temperature $\left(310^{\circ} \mathrm{K}\right)$

\begin{tabular}{lcccc}
\hline Sample & $\begin{array}{c}\text { Sample } \\
\text { thickness } \\
(\mu \mathrm{m})\end{array}$ & $\begin{array}{c}\text { Phase shift } \\
\text { per unit } \\
\text { length (deg.) }\end{array}$ & $\begin{array}{c}\text { Present } \\
\text { work }\end{array}$ & Literature \\
\hline \multirow{3}{*}{ Teflon } & 90 & 0.61 & 2.03 & $2.08^{a}$ \\
& 130 & 0.96 & 2.03 & $2.07 \pm 0.03^{b}$ \\
& 186 & 1.28 & 2.02 & \\
Mylar & 75 & 1.15 & 3.13 & \\
& 130 & 1.91 & 3.10 & $3.145^{c}$ \\
& 250 & 3.74 & 3.18 & \\
\hline
\end{tabular}

' Von Hippel (1961); ' Pilipenko et al (1970); c Conklin (1965).

Table 2. Complex permittivity for a p-type silicon $(5.45 \Omega \mathrm{cm})$ at room temperature $\left(310^{\circ} \mathrm{K}\right)$ and at a frequency of $9.410 \mathrm{GHz}$

\begin{tabular}{|c|c|c|c|c|}
\hline $\begin{array}{l}\text { Thickness } \\
\text { (microns) }\end{array}$ & $\begin{array}{l}\text { Phase shift } \\
\text { per unit } \\
\text { length } \\
\text { (degrees) }\end{array}$ & $\begin{array}{l}\text { Attenuation per } \\
\text { unit length } \\
\text { (decibles) }\end{array}$ & $\varepsilon_{r}$ & $\varepsilon_{i}$ \\
\hline 272 & 150 & 8.6 & 11.61 & $30-58$ \\
\hline 226 & 13.0 & 7.5 & 11.65 & $32 \cdot 12$ \\
\hline 200 & 12.0 & 6.9 & 11.81 & $33 \cdot 13$ \\
\hline 180 & 10.8 & $6 \cdot 1$ & 11.58 & 32.68 \\
\hline 140 & 8.6 & $5 \cdot 1$ & 11.62 & 34.61 \\
\hline 94 & 5.8 & 3.4 & 11.41 & 34.76 \\
\hline 70 & 4.5 & 2.4 & 11.63 & 32.69 \\
\hline
\end{tabular}


Table 3. Comparison of d.c. and a.c. conductivities for three samples of p-silicon

\begin{tabular}{cc}
\hline $\begin{array}{c}\text { d.c. conductivity } \\
\text { by four-probe } \\
\text { technique }(\Omega \mathrm{m})^{-1}\end{array}$ & $\begin{array}{c}\text { Microwave conductivity } \\
\text { from } \sigma_{\text {a.c. }}=\omega \varepsilon_{0} \varepsilon_{i} \\
(\Omega \mathrm{m})^{-1}\end{array}$ \\
\hline 10.26 & 9.79 \\
15.63 & 15.17 \\
18.35 & 17.97 \\
\hline
\end{tabular}

consistant with the usual expressions for a.c. and d.c. conductivities.

and

$$
\sigma_{\text {a.c. }}=\frac{n e^{2} v}{m\left[v^{2}+\omega^{2}\right]},
$$

$$
\sigma_{\text {d.c. }}=\frac{n e^{2}}{m v}
$$

where $m, e, n$ and $v$ are the mass, charge, concentration and collision frequency of the holes in $p$-silicon.

The microwave data may also be used to determine other transport parameters of silicon. The dielectric constant and conductivity in terms of effective mass $m^{*}$ and relaxation time $\tau$ may be written as

$$
\begin{aligned}
& \varepsilon_{r}=\varepsilon_{L}-\frac{n e^{2} \tau^{2}}{\varepsilon_{0} m^{*}\left[1+(\omega \tau)^{2}\right]}, \\
& \sigma=\frac{n e^{2} \tau}{m^{*}\left[1+(\omega \tau)^{2}\right]}=\omega \varepsilon_{0} \varepsilon_{i},
\end{aligned}
$$

where $\varepsilon_{L}$ is the lattice dielectric constant and $\varepsilon_{0}$ is the permittivity of free space. From the above two equations, we get

$$
\tau=\frac{\varepsilon_{L}-\varepsilon_{r}}{\omega \varepsilon_{i}} .
$$

With the present results the relaxation time in silicon comes out to be around $10^{-13} \mathrm{sec}$, which agrees well with the data $\left(\simeq 10^{-13} \mathrm{sec}\right)$ produced by Blatt $(1968)$.

The conductivity variations with temperature for $p$-silicon samples (d.c. resistivities $10 \cdot 5,40$ and $170 \mathrm{ohm} \mathrm{cm}$ ) in the temperature range $190-525^{\circ} \mathrm{K}$ are plotted in figure 6. The curves for the three silicon samples exhibit broadly similar characteristics. viz the conductivity initially decreases with increase of temperature and later increases very rapidly with temperature. This is, of course, expected, considering the relative predominance of extrinsic and intrinsic conduction mechanisms. The conductivity of the samples (having resistivities 10.5 and $40 \mathrm{ohm}-\mathrm{cm}$ ) decreases from room temperature to $440^{\circ} \mathrm{K}$. In this temperature region lattice scattering by acoustical phonons plays the dominant role (Blackmore 1969). As the temperature is raised further, the concentration of free electrons and holes increases and the intrinsic conduction predominates thereafter. In the case of the third sample $(170 \mathrm{ohm} \mathrm{cm}$, the highest resistivity of all the 


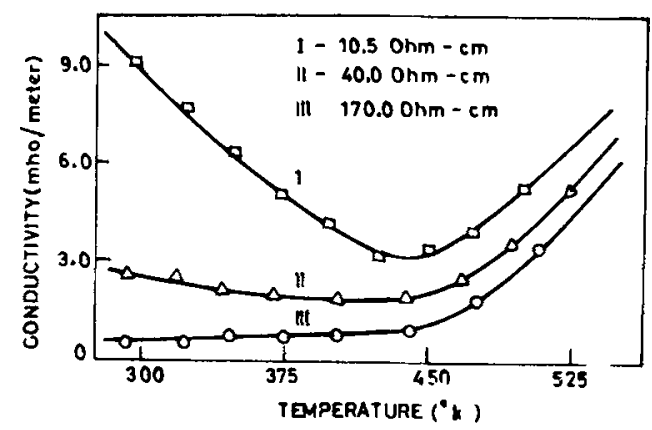

Figure 6. Microwave conductivity variation of silicon wafers in the temperature range $293-523^{\circ} \mathrm{K}$.

three) the conductivity rises slowly from room temperature up to $440 \mathrm{~K}$ and increases very rapidly subsequently thereby showing its intrinsic behaviour right from room temperature. Bhar (1963) made similar measurements on temperature variations of microwave conductivity for bulk $p$-silicon samples having conductivities 0.7 and $0.1 \mathrm{mho} \mathrm{m}^{-1}$ in the temperature range $273-480^{\circ} \mathrm{K}$. His results also resemble the curves of figure 6.

Electrical parameters have been measured for metal and semimetal films of thicknesses less than the skin depths deposited on glass substrates by vacuum thermal evaporation technique (Dube et al 1978).

Figures 7 and 8 show the phase shift and attenuation constants as a function of film thickness for the semimetal bismuth. Table 4 contains the results for $\varepsilon_{r}$ and $\varepsilon_{i}$ for bismuth films of different thicknesses. The high values of $\varepsilon_{r}$ and $\varepsilon_{i}$ are attributed to the presence of free carriers and are consistent with the results of Rahneberg (1971) for bismuth films measured by a different technique at $14.50 \mathrm{GHz}$. Gold and aluminium films also show high dielectric parameters of the order of $10^{5}$ (Dube et al 1978).

Dielectric behaviour of pure polyvinyl chloride (PVC) and iodine-doped PVC has been studied at microwave frequencies using the proposed technique (Natarajan and Dube 1981). Pure PVC films were obtained by dissolving the polymer powder in tetrahydrofuran and permitting the evaporation of the solvent at room temperature. Iodinedoped PVC samples were prepared from the Pvc solution in which a requisite amount of iodine was dissolved. The concentration of jodine in the samples varied from $4-40 \%$ by weight.

Figure 9 depicts the variation of $\varepsilon_{r}$ with temperature for all PvC samples. It is seen that at any fixed temperature, the dielectric constant increases as the iodine content in the sample increases. This effect is more pronounced for iodine concentrations above $\sim 5 \%$ (by weight). For temperatures above $90^{\circ} \mathrm{C}$ the dielectric constant decreases for all samples containing iodine.

The rise in $\varepsilon_{r}$ with iodine concentration may be attributed to the presence of highly polarizable molecular iodine in the specimen. The presence of free iodine was detected in the absorption spectrum in the visible region.

The volume expansion due to transition from glassy to rubbery state seems to account for the decrease of $\varepsilon_{r}$ with temperature in the vicinity of $85^{\circ} \mathrm{C}$ as the glass transition temperature $\left(T_{g}\right)$ of PVC falls in the region $70-105^{\circ} \mathrm{C}$ (Brandrup and Immergut 1975). Variation of loss-tangent $(\tan \delta)$ with temperature for different iodine 


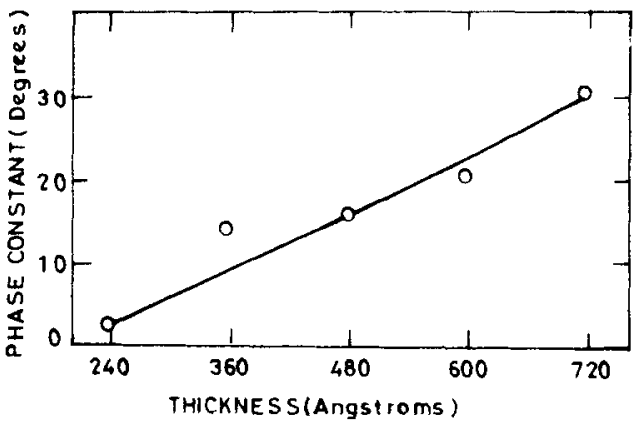

Figare 7. Variation of phase constant as a function of bismuth film thickness.

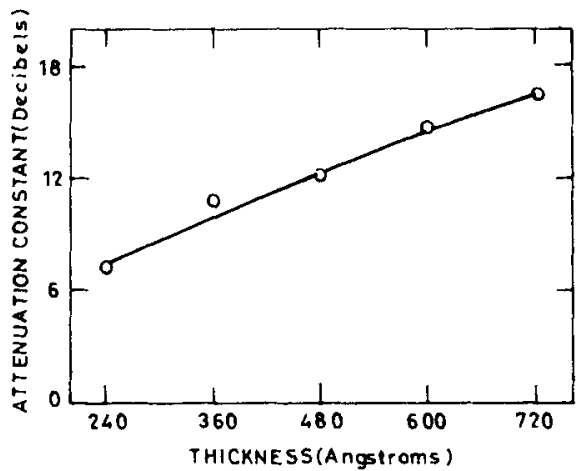

Figure 8. Variation of attenuation constant as a function of bismuth film thickness.

Table 4. Complex permittivity for thin bismuth films for various thicknesses at $310^{\circ} \mathrm{K}$ and at a frequency of $9 \cdot 410 \mathrm{GHz}$.

\begin{tabular}{lcccc}
\hline $\begin{array}{l}\text { Thickness } \\
\text { (angstroms) }\end{array}$ & $\begin{array}{c}\text { Phase shift/ } \\
\text { length } \\
\text { (degrees) }\end{array}$ & $\begin{array}{c}\text { Attenuation/ } \\
\text { length } \\
\text { (decibels) }\end{array}$ & $\begin{array}{c}\text { Dielectric parameters }\left(\times 10^{5}\right) \\
\varepsilon_{r}\end{array}$ & $\varepsilon_{i}$ \\
\hline 240 & 2.5 & 7.5 & 0.36 & 3.12 \\
360 & 12.0 & 10.8 & 0.91 & 2.91 \\
480 & 15.5 & 12.2 & 0.89 & 2.38 \\
600 & 20.0 & 13.7 & 0.83 & 1.83 \\
720 & 30.0 & 16.3 & 1.05 & 1.86 \\
\hline
\end{tabular}

dopings has been shown in figure 10 . For iodine content of $10 \%$ and above, welldefined loss-peak appears at around $90^{\circ} \mathrm{C}$ in each case. With increasing iodine content, the position of loss peak does not change; however, the peak height changes considerably. The fact that loss tangent maxima does not change in position but only in magnitude with iodine concentration shows that on increasing the doping the number of dipoles participating in the orientation process increases, while the frequency of orientation remains unchanged. 


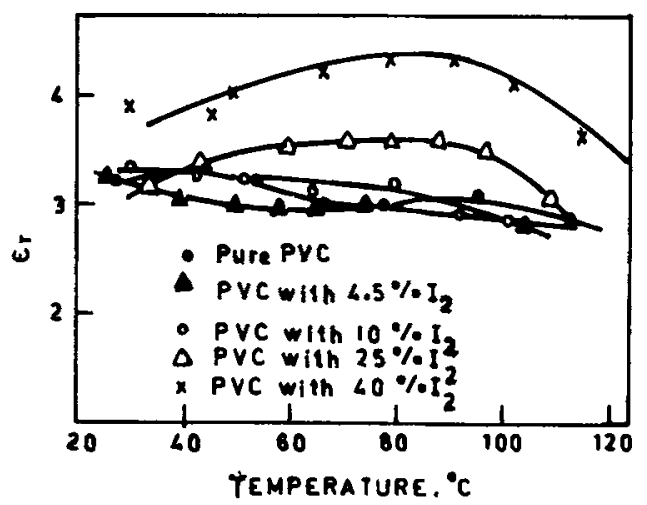

Figure 9. Variation of $\varepsilon_{r}$ with temperature for pure and iodine doped PVc.

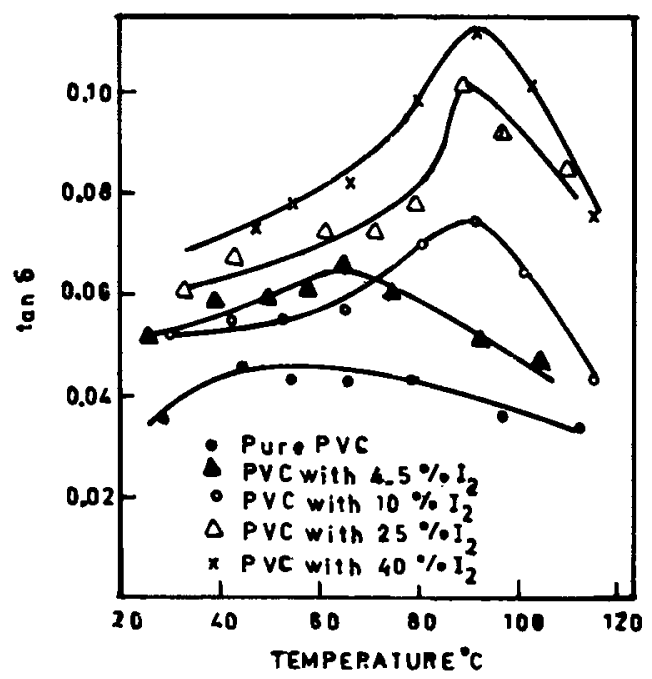

Figure 10. Variation of $\tan \delta$ with temperature for pure and iodine doped Pvc.

Fast ion conducting solids (also called superionic conductors) have become very important in the recent past due to their applications in many electrochemical devices. Electrical measurements on these materials require special attention due to the variety of electrodes (blocking, reversible and their combinations) (Bottelberghs 1978) used making the analysis more complex. The ionic conduction has been studied recently at microwave frequencies (Dube and Saraswat 1982) in silver iodide in the high conductivity $\alpha$-phase. Thin pellets of AgI of varying packing density were used and dielectric and conductivity data obtained. This data can be transformed to $100 \%$ dense bulk using correlation formulae (Landau and Lifshitz 1960; Leoyenga 1965; Dube 1970).

$$
\begin{aligned}
& \varepsilon_{p}^{\prime 1 / 3}-1=\delta\left(\varepsilon_{b}^{\prime 1 / 3}-1\right) \\
& \varepsilon_{b}^{\prime \prime}=\frac{\varepsilon_{p}^{\prime \prime}}{\delta}\left(\varepsilon_{b}^{\prime} / \varepsilon_{p}^{\prime}\right)^{2 / 3}
\end{aligned}
$$


Table 5. Conductivity and dielectric constant for $\alpha-\mathrm{AgI}\left(T=160^{\circ} \mathrm{C}\right)$ at $10 \mathrm{kHz}$

\begin{tabular}{|c|c|c|c|c|c|c|}
\hline \multirow{2}{*}{$\begin{array}{c}\text { Packing } \\
\text { fraction } \\
\%\end{array}$} & \multicolumn{2}{|c|}{$\begin{array}{l}\text { Observed parameters } \\
\text { for pellets }\end{array}$} & \multicolumn{2}{|c|}{$\begin{array}{l}\text { Values converted } \\
\text { to } 100 \% \text { density by } \\
\text { correlation formulae }\end{array}$} & \multicolumn{2}{|c|}{$\begin{array}{l}\text { Values reported } \\
\text { in literature in } \\
\text { microwave region } \\
\text { (Gebhardt et al } 1980 \text { ) }\end{array}$} \\
\hline & $\begin{array}{l}\text { Dielectric } \\
\text { constant }\end{array}$ & $\begin{array}{l}\text { Conductivity } \\
(\Omega \mathrm{cm})^{-1}\end{array}$ & $\begin{array}{l}\text { Dielectric } \\
\text { constant }\end{array}$ & $\begin{array}{l}\text { Conductivity } \\
(\Omega \mathrm{cm})^{-1}\end{array}$ & $\begin{array}{l}\text { Dielectric } \\
\text { constant }\end{array}$ & $\begin{array}{l}\text { Conductivity } \\
(\Omega \mathrm{cm})^{-1}\end{array}$ \\
\hline 72 & $12 \cdot 0$ & 0.044 & $21 \cdot 74$ & 0.092 & \multirow{4}{*}{$22 \cdot 00$} & \multirow{4}{*}{1.22} \\
\hline 82 & 15.4 & 0.076 & $22 \cdot 30$ & 0.119 & & \\
\hline 86 & 16.9 & 0.084 & 22.45 & $0 \cdot 110$ & & \\
\hline 92 & 19.4 & 0.088 & 22.75 & $0 \cdot 116$ & & \\
\hline
\end{tabular}

and

$$
\sigma_{b}=\omega \varepsilon_{0} \varepsilon_{b}^{\prime \prime},
$$

where $\varepsilon_{p}^{\prime}$ and $\varepsilon_{p}^{\prime \prime}$ are the dielectric constant and loss factor for the pellet of packing fraction $\delta$ and $\varepsilon_{b}^{\prime}, \varepsilon_{b}^{\prime \prime}$ are the corresponding parameters for the bulk. $\sigma_{b}$ is the conductivity at angular frequency $\omega . \varepsilon_{0}$ is permittivity of free space $(=8.85$ $\times 10^{-12} \mathrm{Fm}^{-1}$ ). Various results have been summarised in table 5 . It is seen that while the deduced dielectric constant data match with experimentally reported value of 22 , the conductivity results are found reduced by an order of magnitude. This reduction in conductivity is attributed to the presence of grain boundaries in the unsintered samples used in the above studies.

In conclusion, it may be said that the microwave technique described is very convenient and accurate and can be used to study electrical transport behaviour of materials differing widely in characteristics.

\section{References}

Bhar J N 1963 Proc. IEEE 511623

Blakemore J S 1969 Solid State Physics (Toronto: Saunders Company)

Blatt F J 1968 Physics of electronic conduction in solids (New York: McGraw-Hill)

Bottelberghs P H 1978 in Solid electrolytes: General principles, characterization, materials, applications (eds)

Paul Hagenmuller and W Van Gool (New York: Academic Press)

Brandrup J and Immergut E M (eds) 1975 Polymer handbook (New York: John Wiley) 2nd edn p. 44

Collin R E 1960 Field theory of guided waves (New York: McGraw-Hill) Chap. 6

Conklin G E 1965 Rev. Sci. Instrum. 361347

D'Aiello R V and Freedman S J 1969 J. Appl. Phys. 402156

Das Gupta C and Pramanick P 1982 Microwave 76

Dube D C 1970 J. Phys. D3 1648

Dube D C and Natarajan R 1973 J. Appl. Phys. 444927

Dube D C and Natarajan R 1974 J. Phy's. E7 256

Dube D C, Natarajan R and Tripathi V K 1978 Indian J. Phys. A52 405

Dube D C and Saraswat A K 1982 J. Appl. Phys. 537095

Duggal V P and Rup R 1969 J. Appl. Phys. 40492

Gebhardt K F, Soper P D, Merski J, Balle T J and Flygare W H 1980 J. Chem. Phys. 72272

Gunn M W 1967 IEEE Proc. 114207

Korolev F A and Gridnev V I 1963 Radiotekh. Electron. 81480 
Landau L D and Lifshitz F M 1960 Electrodynamics of continuous media (London: Pergamon) p. 46 Looyenga H 1965 Physica 31401

Natarajan R, Dube D C and Tripathi V K 1978 Indian J. Phys. 52567

Natarajan R and Dube D C 1981 Indian J. Pure Appl. Phys. 19675

Pilipenko V V, Polovnikov G G, Sologub V G and Shestopilov V P 1970 Sov. Phys. Tech. Phys. 141662

Rahneberg E W 1971 Measurement of the Complex Permittivity of Thin Bismuth Films within the Ku-band Ph.D. Thesis, University of Arizona USA

Ramey R L, Landes H S and Manus E A 1970 IEEE Trans. Microwave Theory Technol. MTT-18 196

Rzepecka M A and Hamid M A K 1972 IEEE Trans. Microwave Technol. MTT-20 30

Sobol H and Hughes J J 1967 IEEE Trans. Microwave Technol. MTT-15 377

Von Hippel A R 1961 Dielectric materials and applications (New York: Wiley) 